\title{
Cognitive Radio Protocols Based on Exploiting Hybrid ARQ Retransmissions
}

\author{
Ramy Abdallah Tannious and Aria Nosratinia, Fellow, IEEE
}

\begin{abstract}
This paper addresses user coexistence in cognitive radio systems by taking advantage of opportunities that arise during ARQ retransmission. It is shown that if these opportunities are properly exploited, nontrivial rates can be made available to a secondary (cognitive) pair while impinging little or no interference on the primary pair. This can be accomplished with an oblivious primary system and without assuming any non-causal information at the secondary about the primary data. Several protocols are devised that work with varying amounts of channel state information about the cognitive and primary links. The protocols are further extended to the scenario where multiple cognitive receivers exist. Performance analysis of the protocols is presented and their effectiveness is verified via simulations.
\end{abstract}

Index Terms-Cognitive radio, coexistence, fading channel, hybrid ARQ, outage probability, power control.

\section{NOMENCLATURE}

$\mathbb{P}\{\cdot\} \quad$ Probability of an event

$C_{p}, C_{s} \quad$ Primary and secondary throughput

$C_{\text {ref }} \quad$ Throughput of benchmark (primary-only) system

$R_{p}, R_{s} \quad$ Primary and secondary attempted rate

$P_{p}, P_{s} \quad$ Primary and secondary average power

$P_{p c} \quad$ Secondary instantaneous power with power control

$O_{p}, O_{s} \quad$ Primary and secondary outage events

$O_{\text {ref }} \quad$ Outage event of benchmark system (primary-only)

$O_{i n i} \quad$ Outage of primary receiver after one transmission

$S \quad$ Event: secondary Rx decodes primary's 1st packet

$U \quad$ Event: secondary link is activated

$h_{i, j} \quad$ Channel gain between transmitter $i$ and receiver $j$

SNR Average SNR of a link

$\hat{\gamma} \quad$ Secondary's estimate of primary received power

\section{INTRODUCTION}

C OGNITIVE radios promise efficient use of the underutilized, yet scarce, wireless spectrum [1], [2]. The basic requirement of a cognitive radio is to be aware of the primary users in the environment and impinge no more than minimal interference on them. The theoretical and practical problems involved in cognitive radio have proved to be very challenging.

Manuscript received August 1, 2009; revised December 27, 2009; accepted March 13, 2010. The associate editor coordinating the review of this paper and approving it for publication was E. Hossain.

R. A. Tannious is with the Department of Electrical and Computer Engineering, University of California, Davis, CA, 95616, USA (e-mail: rtannious@ucdavis.edu).

A. Nosratinia is with the Department of Electrical Engineering, The University of Texas at Dallas, Richardson, TX (e-mail: aria@utdallas.edu).

This work was done while the first author was with the University of Texas at Dallas. This research is supported in part by the National Science Foundation (NSF) grant CNS-0435429 and in part by the grant 009741-00842007 from Texas Higher Education Coordinating Board (THECB).

Digital Object Identifier 10.1109/TWC.2010.062910.091162

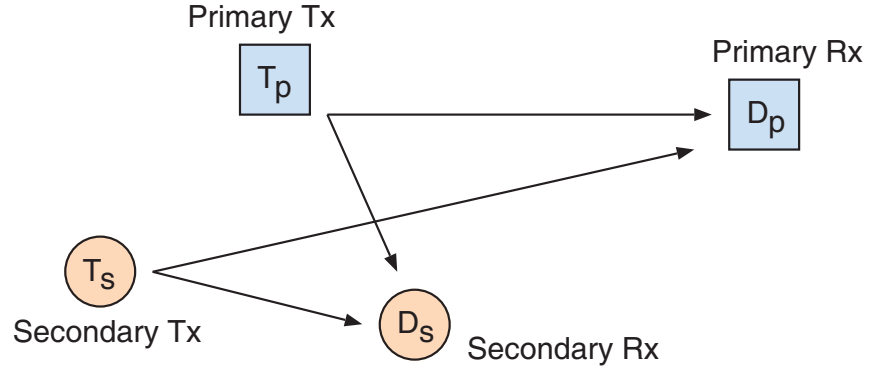

Fig. 1. Cognitive radio channel model.

A recent summary of results in dynamic spectrum sharing is offered in [3].

The past decade of work in cognitive radio has taught us that acquiring knowledge of primary users and their activity can be challenging. This in turn suggests that any available knowledge about the structure of the primary transmissions is precious and must be carefully exploited. Following this general philosophy, this paper exploits the primary hybridARQ retransmissions to simultaneously furnish nontrivial rates for a secondary pair that coexists with the primary pair while impinging minimal interference on the primary receiver. The proposed methods aim to maintain practicality (e.g. no noncausal knowledge of data or channels is assumed) while staying true to the fundamental ideas of cognitive radio (oblivious primary system, minimal or non-existent secondaryon-primary interference).

To shed further light on the basic ideas of this paper, we provide a simple toy example using a cognitive channel model of Fig. 1. We plot the mutual information carried via one or two rounds of transmissions through the primary link (see Fig. 2). The horizontal axis shows the effective receive SNR for the primary link. It is assumed that the desired primary transmission rate is $R_{p}=3$ bits per channel use. For a range of receive SNR, the first transmission does not provide enough mutual information and therefore a second (ARQ) transmission will take place. In most of these cases, however, the mutual information provided by two transmissions is in fact more generous than necessary. Broadly speaking, this "excess" mutual information means that the link can tolerate extra noise/interference. The protocols in this paper exploit this under-utilization of resources during ARQ transmissions.

It is notable that even though ARQ retransmissions may be relatively infrequent, it is still possible to extract attractive average rates for the secondary link, but this requires careful management of channel resources. For example, the secondary receiver listens to the first round of the primary transmissions, 


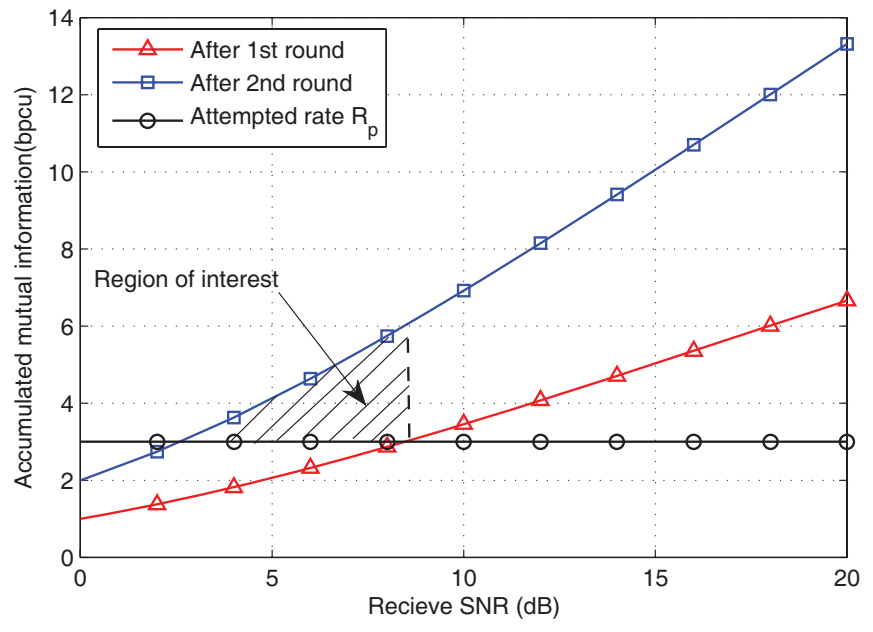

Fig. 2. Mutual information after first and second rounds.

so that during the re-transmissions it can cancel the interference caused by the primary. Experiments will show that interference cancellation by the secondary as well as careful resource management will indeed give rise to nontrivial rates for the secondary system while impinging minimal or no adverse effect on the primary pair.

We now briefly outline some of the related work in the literature. Research in cognitive radios initially concentrated on sensing nearby transmissions and identifying spectrum holes, also known as "white spaces." Although interesting progress has been made in opportunistic spectrum sensing, there is some evidence [4] that it may confront some fundamental limitations. The information theory community approached the problem as a modified form of the interference channel [5], via a model known as the cognitive radio channel [6]. Many of the works in this area concentrate on the Gaussian channel and assume a genie-aided secondary that has non-causal access to the primary's data. A recent survey of these efforts appears in [7].

Gastpar [8] formulated the problem of capacity under receive signal constraints in AWGN channels. The work in [9] investigates the limits of communication in ergodic fading channels subject to peak or average interference constraint on a third-party receiver. It is shown that fading variations can lead to much higher capacity compared to the AWGN case. This work was extended in [10] to the ergodic, outage and minimal-rate capacities of a Rayleigh point-to-point channel under joint peak and average received-power constraints at a third-party receiver.

Chen et al. [11] considers power control in a block-fading model to allow cognitive users to coexist with primary user without affecting the primary's outage probability. This work requires instantaneous channel state information of all links at the secondary transmitter. The work in [12] has some similarities to our work. However, it assumes an ergodic fading setup in contrast to our quasi-static fading model and hence assumes power control across the fading states per transmission. Also, [12] assumes the existence of perfect CSI about the primary transmitter-to-receiver channel and focuses on finding optimal power control policies for the secondary user that minimize the ergodic capacity loss of the primary receiver. The subsequent work of Zhang et al. [13] considers also an ergodic fading setup but studies power control policies for secondary multiple-access and broadcast channels. Koyluoglu and El Gamal [14] studies power control in the context of a generalized cognitive radio model, which allows coexistence under both active and idle periods of the primary user transmission. It is shown that multiple antennas at the secondary transmitter and receiver provide impressive throughput gains during the active period of primary transmission. The advantages of multiple antennas for opportunistic spectrum sharing are thoroughly studied in [15]. In [16], the primary link is modeled as an erasure channel and simple ARQ is used for retransmission of erased packets. A cognitive user exploits the fact that it can eavesdrop on the handshaking signal of the primary's ARQ to adaptively adjust its input in a discrete memoryless channel such that the primary user achieves its target rate.

The rest of this paper is organized as follows. Section II describes the system model, and Section III presents outage and throughput analysis in the absence of CSI at the secondary. Section IV develops a relaying-based protocol in the presence of CSI that guarantees zero interference. To relax the CSI requirements, Section $\mathrm{V}$ develops protocols that require only partial CSI at the secondary transmitter. This class of protocols is extended to a scenario involving multiple secondary receivers. We verify the validity of the proposed protocols by extensive simulations and present numerical results and related discussions in Section VI. Finally, Section VII concludes the paper.

\section{System Model and Problem Statement}

A summary of our most frequently used notations appears in the table at the beginning of this paper. Throughout the paper, lower-case boldface letters denote vectors. Probabilistic events are shown with upper case letters, e.g., $A$, and their complement are denoted with a bar, e.g., $\bar{A}$. All logarithms are in base- 2 .

We consider a network with a pair of primary transmitter $T_{p}$ and receiver $D_{p}$. The secondary (cognitive) transmitter $T_{s}$ and receiver $D_{s}$, as depicted in Fig. 1. It is assumed that the secondary pair communicates at the same frequency band and time slot allocated to the primary pair.

The channel gain between any two nodes is described by a flat, quasi-static Rayleigh fading model. Thus, the channel is constant within a coherence interval and changes independently from one coherence interval to another. Whenever a retransmission occurs, it is assumed that the channel remains constant across ARQ rounds. The receivers are assumed to have perfect receive channel state information (CSI) allowing coherent detection. The channel gains are denoted with $h_{i, j}$, where $i$ and $j$ are the indices of the transmitter and receiver, respectively. The index 1 is reserved for the primary transmitter and receiver, and indices 2 and higher belong to secondary nodes. The magnitude square of $h_{i, j}$, also known as effective channel gain, follows an exponential distribution. The thermal noises at the receivers are assumed additive to the incoming signals and are normally distributed $\sim \mathcal{N}\left(0, \sigma^{2}\right)$. Without loss of generality, it is assumed that $\sigma^{2}=1$ for all nodes. 
The primary and secondary transmitters operate under an average per-block power constraint $P_{p}$ and $P_{s}$, respectively. We assume all codewords are drawn from a Gaussian codebook. The codewords fit within a coherence interval but are sufficiently large to allow reliable decoding whenever the mutual information of the channel supports the attempted rate. All nodes are half-duplex, i.e. cannot transmit and receive at the same time/frequency. It is assumed that the primary user transmits at a fixed power $P_{p}$, fixed nominal rate $R_{p}$, and employs hybrid ARQ with incremental redundancy (IR) with a limit of one retransmission. The limit of one retransmission captures most of the gains of HARQ and limits the delay in decoding. The secondary transmitter on the other hand can transmit at fixed or variable power as it will be described in the sequel, but employs no rate control. It is assumed that the secondary pair knows the codebook used by the primary transmitter.

The system model so far resembles that of an interference channel. However, in a cognitive radio setup, there are further restrictions on the abilities and actions of primary and secondary users, namely:

1) Little or no rate loss is experienced at the primary receiver due to the communication between the secondary nodes.

2) The primary nodes are oblivious to the presence of the secondary nodes. Thus, the primary nodes do not change their encoding or decoding strategy according to the presence of the secondary. The primary receiver uses a single-user decoding strategy.

The goal of this paper is to devise protocols that satisfy the above requirements and yet do not depend on impractical assumptions. The performance of the proposed protocols is measured by outage [17] and expected throughput [18]. Outage is the event that the mutual information across the channel is less than the attempted rate $R$. Assume the received signal at node $j$ is given by:

$$
\mathbf{y}_{j}=\sqrt{\mathrm{SNR}} h_{i, j} \mathbf{x}_{i}+\mathbf{z}_{j}
$$

where $\mathbf{x}_{i}$ is the transmitted codeword by node $i$ and $\mathbf{z}_{j}$ is the AWGN. Conditioned on $h_{i, j}$ at the receiver, the probability of outage is given by:

$$
P_{\text {out }}=\mathbb{P}\left\{\log \left(1+\left|h_{i, j}\right|^{2} \mathrm{SNR}\right)<R\right\}
$$

The corresponding expected throughput is given by:

$$
C=R\left(1-P_{\text {out }}\right)
$$

\section{Signaling StRategy With No TRANSMit-Side CSI}

\section{A. Protocol Description}

We assume that the secondary transmitter has no CSI except overhearing the ACK(NACK) signal transmitted by the primary receiver at the end of the initial round of transmission. The protocol works as follows:

1) During time interval $b$, the primary node $T_{p}$ transmits a packet while both primary and secondary receivers listen. If the primary receiver $D_{p}$ successfully decodes, it emits an ACK. Upon hearing the ACK, $D_{s}$ will discard its received packet and $T_{p}$ gets ready to send a new message packet.

2) If the primary receiver is not able to decode, it will emit a NACK. This will be heard by the secondary receiver $D_{s}$, who will then attempt to decode the primary signal that it has stored in its buffer. If successful, $D_{s}$ will send a "Clear to Send" (CTS) signal to $T_{s}$.

3) When a primary NACK has been issued, in block $b+1$ the primary $T_{p}$ retransmits its packet. If furthermore a secondary CTS has been generated, $T_{s}$ transmits its cognitive message simultaneously. The secondary receiver can fully peel off the primary interference from its received signal since it already knows the primary message. The primary receiver combines the received codewords from blocks $b$ and $b+1$.

4) Both receivers attempt decoding their respective messages. Each receiver, upon failure, will declare outage. Thus, the primary user has up to two rounds of transmissions while the secondary user in this scenario is allowed only one transmission.

The secondary transmission produces interference on the primary during the retransmission period, which will be analyzed in the sequel. Strategies will be considered to eliminate or minimize the effect of this interference.

The key features of this protocol are:

- The secondary pair communicates only during ARQ rounds of the primary.

- The secondary receiver uses a simple interference cancellation scheme in order to get a clean signal for its message.

- In cases where the primary-on-secondary interference cannot be canceled, the secondary pair remains silent. This is motivated by the idea that the operation of the secondary pair has costs (interference on primary), therefore the secondary link should only be active when it can harvest good rates, otherwise it should stay silent.

\section{B. Outage and Throughput Analysis}

We start by analyzing the outage of the primary user while the secondary user is absent, to be used as a benchmark for the performance of the primary under coexistence by the secondary.

Since there is only one ARQ transmission, outage occurs in the primary link if the accumulated mutual information from the two transmissions is less than the attempted rate $R_{p}$. Let $O_{\text {ref }}$ denotes the outage event of the primary user, then ${ }^{1}$

$$
\begin{aligned}
\mathbb{P}\left\{O_{\text {ref }}\right\} & =\mathbb{P}\left\{2 \log \left(1+\left|h_{1,1}\right|^{2} P_{p}\right)<R_{p}\right\} \\
& =1-\exp \left(-\frac{2^{\frac{R_{p}}{2}}-1}{\lambda_{p} P_{p}}\right)
\end{aligned}
$$

Where $\lambda_{p}$ is the mean of the channel $h_{1,1}$. Define $O_{i n i}$ to be the event describing the failure of the initial transmission by

\footnotetext{
${ }^{1}$ The reader is reminded that we assume the channels remain constant across the initial and retransmission rounds.
} 
the primary user. The probability of this event is given by:

$$
\begin{aligned}
\mathbb{P}\left\{O_{i n i}\right\} & =\mathbb{P}\left\{\log \left(1+\left|h_{1,1}\right|^{2} P_{p}\right)<R_{p}\right\} \\
& =1-\exp \left(-\frac{2^{R_{p}}-1}{\lambda_{p} P_{p}}\right)
\end{aligned}
$$

The corresponding expected throughput depends on whether a packet is received in one try, or two tries and is given by:

$$
\begin{aligned}
C_{\text {ref }} & =R_{p} \mathbb{P}\left\{\bar{O}_{i n i}\right\}+\frac{R_{p}}{2} \mathbb{P}\left\{\bar{O}_{r e f} \mid O_{i n i}\right\} \\
& =R_{p}\left(1-\mathbb{P}\left\{O_{i n i}\right\}\right)+\frac{R_{p}}{2} \times \\
& \mathbb{P}\left\{\log \left(1+\left|h_{1,1}\right|^{2} P_{p}\right)<R_{p} \leq 2 \log \left(1+\left|h_{1,1}\right|^{2} P_{p}\right)\right\}
\end{aligned}
$$

We now analyze the outage performance of the coexistence protocol described above. Define $O_{p}$ and $O_{s}$ to be the outage event of the primary and secondary receivers, respectively. $S$ is the event that secondary receiver successfully decodes the primary transmission, while $U$ is the event that the secondary link is activated, which is equivalent to $O_{i n i} \cap S$.

$$
\begin{aligned}
\mathbb{P}\{U\} & =\mathbb{P}\left\{\log \left(1+\left|h_{1,1}\right|^{2} P_{p}\right)<R_{p}\right\} \\
& \times \mathbb{P}\left\{\log \left(1+\left|h_{1,2}\right|^{2} P_{p}\right) \geq R_{p}\right\}
\end{aligned}
$$

Using the law of total probability, the outage probability of the primary user is

$$
\mathbb{P}\left\{O_{p}\right\}=\mathbb{P}\left\{O_{p} \mid U\right\} \mathbb{P}\{U\}+\mathbb{P}\left\{O_{p} \mid \bar{U}\right\} \mathbb{P}\{\bar{U}\}
$$

We start by analyzing the first term on the right hand side of (8).

$$
\begin{aligned}
& \mathbb{P}\left\{O_{p} \mid U\right\} \mathbb{P}\{U\}= \mathbb{P}\left\{O_{p} \mid O_{\text {ini }}, S\right\} \mathbb{P}\left\{O_{i n i}, S\right\} \\
&= \mathbb{P}\left\{O_{p}\right\} \mathbb{P}\left\{O_{i n i} \mid O_{p}\right\} \mathbb{P}\left\{S \mid O_{p}\right\} \\
& \stackrel{(a)}{=} \mathbb{P}\left\{O_{p}\right\} \mathbb{P}\left\{S \mid O_{p}\right\} \\
&=\mathbb{P}\left\{O_{p} \mid S\right\} \mathbb{P}\{S\} \\
&=\mathbb{P}\left\{\log \left(1+\left|h_{1,1}\right|^{2} P_{p}\right)\right. \\
&\left.\quad+\log \left(1+\frac{\left|h_{1,1}\right|^{2} P_{p}}{1+\left|h_{2,1}\right|^{2} P_{s}}\right)<R_{p}\right\} \\
& \quad \times \mathbb{P}\left\{\log \left(1+\left|h_{1,2}\right|^{2} P_{p}\right) \geq R_{p}\right\}
\end{aligned}
$$

where $(a)$ is due to the fact that $O_{p}$ implies $O_{i n i}$. Now, we analyze the second term on the right hand side of (8).

$$
\begin{aligned}
\mathbb{P}\left\{O_{p} \mid \bar{U}\right\} \mathbb{P}\{\bar{U}\}= & \mathbb{P}\left\{O_{p}, \bar{U}\right\} \\
= & \mathbb{P}\left\{O_{p},\left(\overline{O_{\text {ini }}, S}\right)\right\} \\
= & \mathbb{P}\left\{O_{p},\left(\overline{O_{\text {ini }}} \cup \bar{S}\right)\right\} \\
\stackrel{(b)}{=} & \mathbb{P}\left\{O_{p}, \bar{S}\right\} \\
= & \mathbb{P}\left\{O_{p} \mid \bar{S}\right\} \mathbb{P}\{\bar{S}\} \\
\stackrel{(c)}{=} & \mathbb{P}\left\{O_{\text {ref }}\right\} \mathbb{P}\{\bar{S}\} \\
= & \mathbb{P}\left\{2 \log \left(1+\left|h_{1,1}\right|^{2} P_{p}\right)<R_{p}\right\} \\
& \quad \times \mathbb{P}\left\{\log \left(1+\left|h_{1,2}\right|^{2} P_{p}\right)<R_{p}\right\}
\end{aligned}
$$

where $(b)$ is due to the fact that $\mathbb{P}\left\{O_{p}, \bar{O}_{i n i}\right\}=0$ and $(c)$ is explained by the fact that if the secondary receiver fails to decode the message of the primary user, the system reduces to the reference system without coexistence. We can now combine Equations (9) and (10) according to (8) to bound the outage probability of the primary system.

Under our protocol, a successful secondary transmission requires a primary NACK, successful decoding of primary by secondary $D_{s}$, and ability of $h_{2,2}$ to support the rate $R_{s}$. Therefore

$$
\mathbb{P}\left\{O_{s}\right\}=1-\left(\mathbb{P}\{U\} \cdot \mathbb{P}\left\{\log \left(1+\left|h_{2,2}\right|^{2} P_{s}\right) \geq R_{s}\right\}\right)
$$

After calculating outage probabilities, we can derive the throughputs. For the primary link:

$$
\begin{aligned}
C_{p}= & R_{p} \mathbb{P}\left\{\bar{O}_{i n i}\right\}+\frac{R_{p}}{2} \mathbb{P}\left\{\bar{O}_{p} \mid O_{i n i}\right\} \\
= & R_{p} \mathbb{P}\left\{\bar{O}_{i n i}\right\} \\
+ & \frac{R_{p}}{2}\left(\mathbb{P}\left\{\bar{O}_{p} \mid O_{i n i}, S\right\} \mathbb{P}\{S\}+\mathbb{P}\left\{\bar{O}_{p} \mid O_{i n i}, \bar{S}\right\} \mathbb{P}\{\bar{S}\}\right) \\
= & R_{p}\left(1-\mathbb{P}\left\{O_{i n i}\right\}\right) \\
+ & \frac{R_{p}}{2}\left(\mathbb { P } \left\{\log \left(1+\left|h_{1,1}\right|^{2} P_{p}\right)<R_{p} \leq \log \left(1+\left|h_{1,1}\right|^{2} P_{p}\right)\right.\right. \\
& \left.+\log \left(1+\frac{\left|h_{1,1}\right|^{2} P_{p}}{1+\left|h_{2,1}\right|^{2} P_{s}}\right\} \mathbb{P}\left\{\log \left(1+\left|h_{1,2}\right|^{2} P_{p}\right) \geq R_{p}\right\}\right) \\
& +\frac{R_{p}}{2}\left(\mathbb{P}\left\{\log \left(1+\left|h_{1,1}\right|^{2} P_{p}\right)<R_{p} \leq 2 \log \left(1+\left|h_{1,1}\right|^{2} P_{p}\right)\right\}\right. \\
& \left.\times \mathbb{P}\left\{\log \left(1+\left|h_{1,2}\right|^{2} P_{p}\right)<R_{p}\right\}\right)
\end{aligned}
$$

The throughput penalty experienced by the primary receiver due to the cognitive transmission is the difference between (6) and (12).

The throughput of the secondary link is simply:

$$
C_{s}=R_{s}\left(1-\mathbb{P}\left\{O_{s}\right\}\right)
$$

\section{FUll CSI AT THE SECONDARY TRANSMITTER}

In the previous section, the secondary transmitter $T_{s}$ did not have channel state information. If CSI can be supplied to $T_{s}$, we can improve the performance via a signaling protocol that is inspired by the decode-forward-dirty-paper coding strategy proposed in [19], [20], [21]. ${ }^{2}$ The performance of a full-CSI protocol can also serve as a point of comparison for other protocols proposed in the sequel that do not use full CSI.

Assume that the secondary transmitter has knowledge about the primary channel $h_{1,1}$ and the cross channel $h_{2,1}$. Moreover, assume that $T_{s}$ decoded successfully the primary message during the initial transmission. This allows $T_{s}$ to relay the primary message through to $D_{p}$ in order to compensate for the interference it is causing to the primary link. Therefore, $T_{s}$ forms the following codeword using the superposition encoding scheme:

$$
\mathbf{x}_{s}=\sqrt{(1-\alpha) P_{s}} \mathbf{x}_{2}+\sqrt{\alpha P_{s}} \frac{h_{2,1}^{*}}{\left|h_{2,1}\right|} e^{j \angle h_{1,1}} \mathbf{x}_{1}
$$

where $\alpha \in[0,1]$. The phase shift introduced in the second term allows coherent addition of the signals at the primary receiver (beamforming).

\footnotetext{
${ }^{2}$ For details and comparisons between different coding strategies for the cognitive radio channel see [7].
} 
The parameter $\alpha$ is obtained as the solution for the following quadratic equation

$\log \left(1+\frac{\left(\left|h_{1,1}\right| \sqrt{P_{p}}+\left|h_{2,1}\right| \sqrt{\alpha P_{s}}\right)^{2}}{1+\left|h_{2,1}\right|^{2}(1-\alpha) P_{s}}\right)=\log \left(1+\left|h_{1,1}\right|^{2} P_{p}\right)$

When a solution exist for $\alpha \in[0,1]$, it is given by

$\alpha=\left(\frac{\left|h_{1,1}\right| \sqrt{P_{p}}\left(1 \pm \sqrt{1+\left|h_{2,1}\right|^{2} P_{s}\left(1+\left|h_{1,1}\right|^{2} P_{p}\right)}\right)}{\left|h_{2,1}\right| \sqrt{P_{s}}\left(1+\left|h_{1,1}\right|^{2} P_{p}\right)}\right)^{2}$

Otherwise, the secondary user remains silent.

The differences between this protocol and the one presented in [21] are summarized as follows:

1) We consider a block-fading channel model and due to channel variations, the analysis cannot be limited to either weak or strong interference, unlike the Gaussian case. This has consequences for the strategy of the secondary user. If a solution for $\alpha$ exists, the secondary uses a relaying scheme with superposition coding. Otherwise it remains silent.

2) The secondary node transmits only during the primary ARQ rounds. Therefore our secondary transmitter has a practical way of acquiring the primary data.

3) Pre-coding at the secondary transmitter is avoided and is replaced by simple interference cancellation at the secondary receiver. This avoids the requirement of knowing $h_{1,2}$ at $T_{s}$ and simplifies signaling. This is noteworthy because it has been shown that pre-coding is quite sensitive to channel phase mismatch [22].

The analysis of outage and expected throughput are along the lines of Section III with the following exceptions. The event $U$ is redefined to include the requirement of the primary message being successfully decoded at $T_{s}$ (in addition to $D_{s}$ ). Moreover, the non-existence of $\alpha \in[0,1]$ causes the secondary transmitter to be silent. The details are omitted for brevity.

\section{POWER CONTROL With PARTIAL TRANSMIT-SIDE CSI}

The previous two sections analyzed the cases of full CSI and no CSI being available at the secondary transmitter. Motivated by the gains of the knowing CSI, but also being aware of the difficulties of imparting full CSI, in this section we devise several protocols that operate under partial CSI at the secondary transmitter.

\section{A. Partial Primary CSI}

By overhearing the ACK/NACK signal, the secondary user can acquire:

1) One bit of information about the channel $h_{1,1}$, i.e. if it is less or more than a certain threshold given the knowledge of $P_{p}$ and $R_{p}$.

2) An estimate of the magnitude of $h_{2,1}$ (the channels are assumed to be reciprocal).

The secondary transmitter uses this information to adapt its power. From the primary's NACK, the secondary transmitter can infer that:

$$
\left|h_{1,1}\right|^{2}<\frac{2^{R_{p}}-1}{P_{p}}
$$

In the absence of exact information, the secondary transmitter $T_{s}$ makes a guess at the received power of the primary; we denote this guess as $\hat{\gamma}$. Because of the NACK, the range of this guess is $0<\hat{\gamma}<2^{R_{p}}-1$. We define a normalized guessing parameter $\eta$ as follows:

$$
\eta \triangleq \frac{\left|h_{1,1}\right|^{2} P_{p}}{2^{R_{p}}-1}
$$

where $0<\eta \leq 1$. The secondary transmitter uses this guess to estimate the impact of its transmission upon the primary link. For example, making a pessimistic guess, i.e. choosing $\eta$ close to zero, will cause the secondary to transmit at lower power, which improves the outage performance of the primary link but causes higher outage probability of the cognitive link.

The power of the secondary transmitter $P_{p c}$ is selected as high as possible while avoiding outage for the primary link, given our guess $\hat{\gamma}$ of its received power. In other words, for the two transmission rounds,

$$
\log (1+\hat{\gamma})+\log \left(1+\frac{\hat{\gamma}}{1+\left|h_{2,1}\right|^{2} P_{p c}}\right) \geq R_{p}
$$

Solving for $P_{p c}$ yields:

$$
P_{p c} \leq \frac{(1+\hat{\gamma})^{2}-2^{R_{p}}}{\left|h_{2,1}\right|^{2}\left(2^{R_{p}}-(1+\hat{\gamma})\right)}
$$

The secondary transmitter also has a per-block power constraint $P_{s}$ which also needs to be honored, therefore

$$
P_{s}^{*}=\min \left(P_{s}, P_{p c}\right)
$$

The outage and throughput analysis follows the previous section with the exception of computing $P_{s}{ }^{*}$ from (21). If a positive solution for $P_{s}{ }^{*}$ does not exist, the secondary user remains silent in that round.

\section{B. Partial Primary CSI Plus Full Cognitive CSI}

We now assume that the cognitive transmitter has CSI, possibly through a feedback link, that carries channel estimates of $h_{2,2}$ from $D_{s}$ to $T_{s}$. We use this information not to enhance the secondary link, but rather to minimize the interference on the primary and reduce the primary outage probability. One may compare and contrast this approach with the use of CSI at the transmitter in single-link point-to-point fixed rate scenarios [23].

We use a truncated channel inversion power control algorithm that is reminiscent of the one discussed in [24] in that our method attempts to cancel channel fading, as long as it is above a certain threshold (cutoff fade depth). However, there are also distinct differences: in [24] the rate is variable and the truncation threshold is optimized for the best overall ergodic capacity, while in our case the attempted rate is fixed and the formulation is non-ergodic.

Specifically, the power control policy is described as follows. The first goal is to compensate for the fading of the cognitive link channel, i.e.,

$$
\log \left(1+P_{s}^{*}\left|h_{2,2}\right|^{2}\right) \geq R_{s}
$$


However, the per-block power constraint as well as the interference constraint on the primary are also in place, similar to the previous section:

$$
P_{s}^{*} \leq \min \left(P_{s}, P_{p c}\right)
$$

Equations (22) and (23) yield:

$$
P_{s}^{*}= \begin{cases}\frac{2^{R_{s}}-1}{\left|h_{2,2}\right|^{2}} & \text { if } \frac{2^{R_{s}}-1}{\left|h_{2,2}\right|^{2}}<\min \left(P_{s}, P_{p c}\right) \\ 0 & \text { otherwise. }\end{cases}
$$

Note that $P_{s}^{*}$ is a function of $h_{2,2}, h_{2,1}$ and $h_{1,1}$. Based on (24), the effect of the cognitive channel knowledge is as follows. The interference seen by the primary receiver is proportional to $\frac{2^{R_{s}}-1}{\left|h_{2,2}\right|^{2}}$ which is always less than $\min \left(P_{s}, P_{p c}\right)$. Therefore, the coexistence penalty decreases. The outage probability of the secondary user on the other hand clearly remains the same as in (21). This is because if $\frac{2^{R_{s}}-1}{\left|h_{2,2}\right|^{2}}>\min \left(P_{s}, P_{p c}\right)$, outage will occur for both power control policies of (21) and (24). On the other hand, if $\frac{2^{R_{s}}-1}{\left|h_{2,2}\right|^{2}}<\min \left(P_{s}, P_{p c}\right)$, outage will not occur as the transmitted power of $\frac{2^{R_{s}}-1}{\left|h_{2,2}\right|^{2}}$ guarantees successful transmission.

We stress again that in contrast to point-to-point channels, we use the secondary CSI at the transmitter to decrease the interference seen by the primary, rather than to improve the performance of the secondary. Although the outage of the secondary link did not change despite knowledge of $h_{2,2}$, there is a subtlety that is worth noting. In our system, the secondary outage stems only from secondary transmitter being silent, which provides power savings. The secondary outage probability is given by

$$
\mathbb{P}\left\{O_{s}\right\}=1-\left(\mathbb{P}\{U\} \cdot \mathbb{P}\left\{\frac{2^{R_{s}}-1}{\left|h_{2,2}\right|^{2}}<\min \left(P_{s}, P_{p c}\right)\right\}\right)
$$

\section{Multiple Secondary Receivers}

We extend the above protocols to multiple secondary users. Consider a secondary broadcast channel, where the secondary transmitter (base station) is interested in communicating independent messages to $K$ secondary users. The secondary transmitter is assumed to possess CSI to its receivers, possibly through a dedicated feedback channel.

It is our goal to investigate the benefits of multiple secondary receivers to the secondary as well as the primary networks. The secondary base station will use the same power control policy described in (24). The only exception is that it transmits to the user with the best channel in order to exploit the multi-user diversity [25]. We adopt an opportunistic transmission scheme that achieves the sum-rate capacity of fading broadcast and multiple-access channels [26]. It is assumed that fixed and equal rate across users is employed (no rate control is used at the transmitter). Thus with enough number of users it is likely that a strong channel exists and outage probability is minimized.

The power of the transmitted signal is given by,

$$
P_{s}^{*}= \begin{cases}\frac{2^{R_{s}}-1}{\left|h_{\max }\right|^{2}} & \text { if } \frac{2^{R_{s}}-1}{\left|h_{\max }\right|^{2}}<\min \left(P_{s}, P_{p c}\right) \\ 0 & \text { otherwise. }\end{cases}
$$

where $h_{\max }$ is the strongest channel among the secondary links, i.e., $\left|h_{\max }\right|=\max _{k}\left|h_{2, k}\right|$. As a result, the existence of $\mathrm{K}$ secondary users provides the following advantages:

1) Increasing the probability that at least one secondary node will decode the message of the primary user. Let this event denoted by $V$, then

$$
\begin{aligned}
\mathbb{P}\{V\} & =1-\prod_{j=2}^{K+1} \mathbb{P}\left\{\log \left(1+\left|h_{1, j}\right|^{2} P_{p}\right)<R_{p}\right\} \\
& =1-\left(1-\exp \left(-\frac{2^{R_{p}}-1}{\lambda_{c} \mathrm{SNR}}\right)\right)^{K}
\end{aligned}
$$

where $\lambda_{c}$ is the mean of the channel between the primary transmitter and a secondary receiver, assumed identical for all secondary users. Therefore, as $K \rightarrow \infty, \mathbb{P}\{V\} \rightarrow$ 1.

2) Similarly if $K \rightarrow \infty$, the outage of the cognitive link will approach zero. Therefore, the outage of secondary transmission will occur in this case only if the primary's first transmission round is successful.

3) Expecting a very good channel at each transmission block and with the truncated channel inversion power control policy leads to lowering the transmission power and hence less interference is impinged on the primary user. Therefore, we expect that the coexistence penalty to diminish which is the goal we strive to achieve in this work.

\section{NumericAl RESUlts AND Discussion}

This section presents numerical results on the performance of the proposed coexistence strategies. The following parameters have been used.

- The effective channel gains are exponentially distributed with unit mean for $\left|h_{1,1}\right|^{2}$ and $\left|h_{2,1}\right|^{2}$ and with mean four for $\left|h_{2,2}\right|^{2},\left|h_{1,2}\right|^{2}$, and $\left|h_{2,1}\right|^{2}$. This models the proximity of the cognitive pair to the primary transmitter compared to the distance between the primary nodes.

- The power of primary user is fixed at $P_{p}=12 \mathrm{~dB}$ and its rate is $R_{p}=3$ bits per channel use (bpcu).

- The attempted rate of the secondary user is tested in the range $0<R_{s} \leq 5$ bpcu.

The results of the protocol with no CSI at the transmitter(Section III) is depicted in Fig. 3, showing the expected throughput versus the attempted rate $R_{s}$. The top three curves in the graph represent the expected throughput of the primary user (for $R_{p}=3 \mathrm{bpcu}$ ) when the secondary nodes are silent or transmitting with $P_{s}=10 \mathrm{~dB}$ and $P_{s}=20 \mathrm{~dB}$. The bottom set of curves show the expected throughput of the secondary pair. Among the latter curves, the top one represents an upper bound derived by assuming that the secondary link is perfect. The other two curves depict the expected throughput with $P_{s}=20 d B$ and $P_{s}=10 d B$.

Figure 4 compares the expected throughput of three protocols proposed in this paper: the protocol with no CSI at the transmitter, the relaying protocol of Section IV, and the partial CSI at the transmitter protocol (Section V-A). The figure confirms that the relaying protocol complies with the strict coexistence constraints and validates the effectiveness of the 


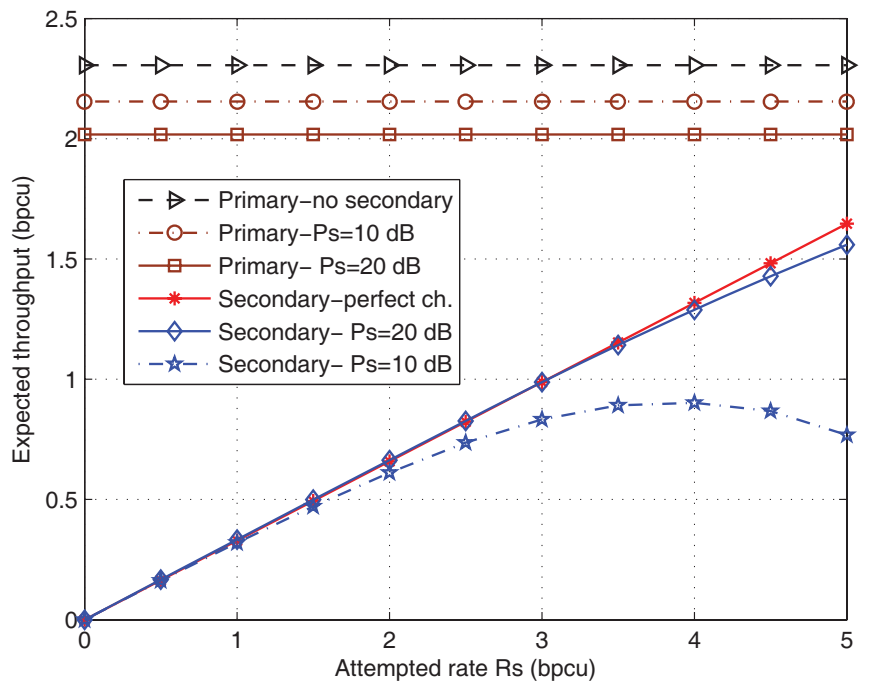

Fig. 3. Expected throughput vs. attempted rate of secondary user $R_{s}$ with no secondary transmitter power control.

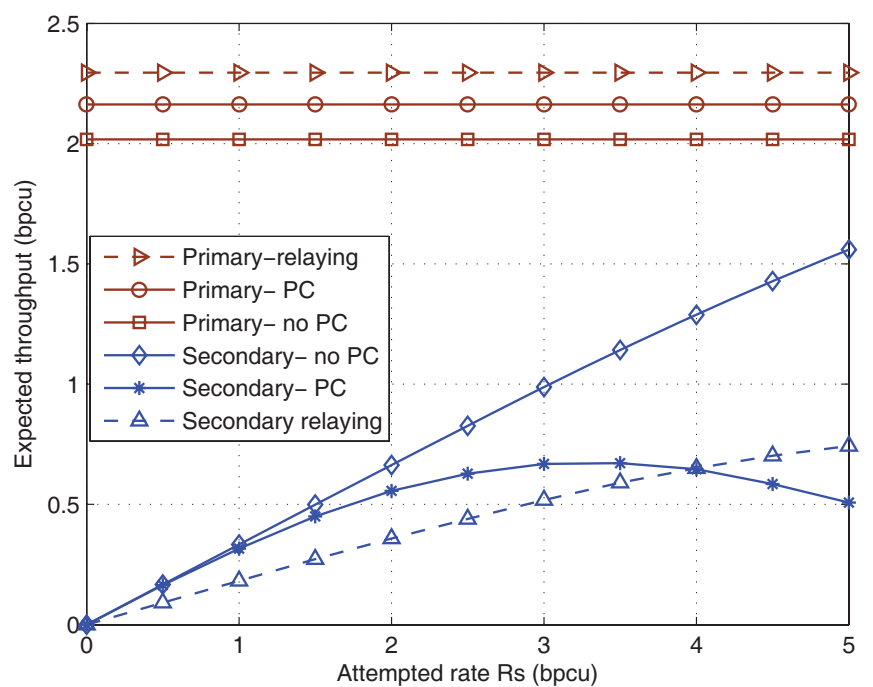

Fig. 4. Comparison of the expected throughput vs. secondary user attempted rate $R_{s}$ with full CSI (relaying protocol), power control with partial CSI, and with no CSI (no power control).

protocol of Section V-A in minimizing the coexistence penalty seen by the primary receiver. It is assumed that $P s=20 \mathrm{~dB}$ and $\eta=0.5$ (see (18)). The relaying protocol compensates fully for the interference and thus the primary user throughput curve is unaffected by the presence of the secondary pair. The simple power control strategy decreases the coexistence penalty on the primary link but entails a loss of throughput for the secondary link. Note that the behavior of the expected throughput curves of the power control strategy depends on the choice of $\eta$. We do not compare the relaying and power control strategies, in part because of the different requirements of the two methods. The former needs the secondary transmitter to decode the primary message.

Figure 5 shows the expected throughput for the secondary and primary users under power control policies of Section V-A and Section V-B. To highlight the ability of cognitive CSI at the transmitter to reduce the coexistence penalty on the primary, we have deliberately increased the penalty on the

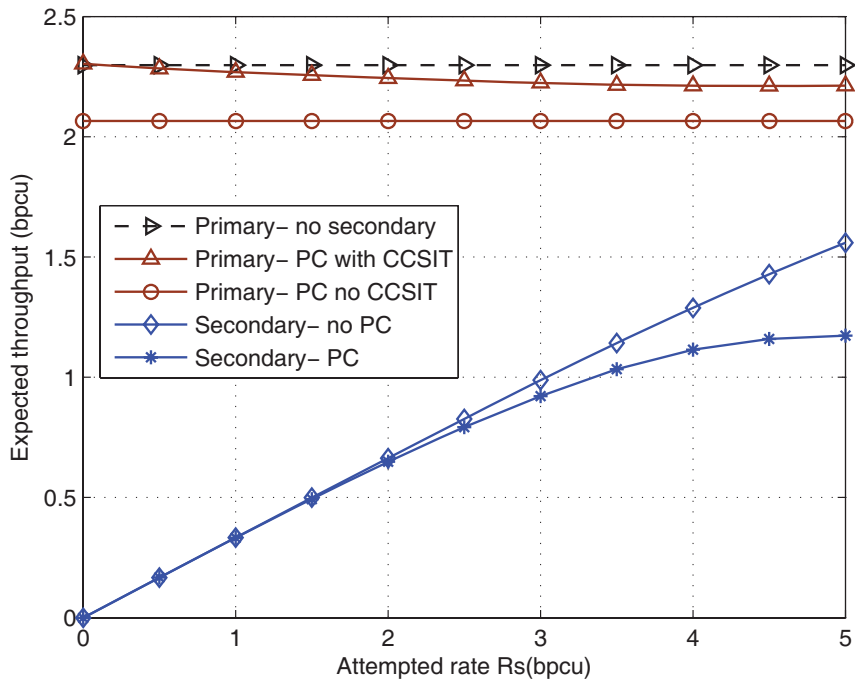

Fig. 5. Effect of CSI of cognitive link (CCSIT) on the expected throughput of primary and secondary users vs. the attempted rate of secondary user $R_{s}$.

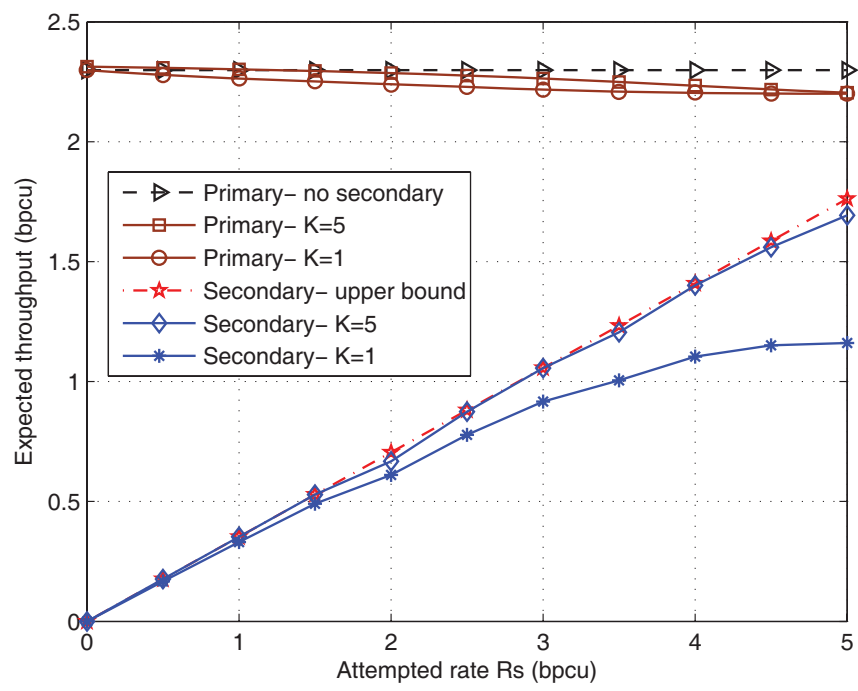

Fig. 6. Expected throughput vs. attempted rate $R_{s}$ with multiple secondary users and availability of CCSIT.

primary by setting $\eta=0.75$. Under this scenario, we observe that the knowledge of the cognitive link CSI at the transmitter can greatly reduce the coexistence penalty on the primary in the low and medium secondary rates $R_{s}$. As mentioned earlier, this protocol has been devised such that all CSI is used to reduce the penalty to the primary, so the secondary performance remains unchanged.

We now verify the claims of Section V-C. We investigate the effect of multiple secondary users to see if the extra degree of freedom enable us to satisfy all the conditions of the problem statement described in Section II. In the experiment of Fig. 6, we have $\eta=0.75$ and also CSI about the cognitive links are available at the cognitive transmitter. The two sets of curves correspond to $K=1$ (identical to the results of Fig. 5) and $K=5$ averaged over the five users. Moreover, we calculate and plot an upper bound on secondary outage performance, namely the probability of success of initial round of primary transmission. It is shown that with only five secondary users in a cognitive downlink network, the coexistence penalty 
virtually disappears across a wide range of $R_{s}$ and the upper bound is almost achieved.

Based on the extensive experiments, we can claim that the elements of the problem statement of this work have been satisfied, namely: a) causing no interference to the primary user, b) achieving non-trivial rates for the secondary user(s). This objective has been achieved with a practical approach that utilizes knowledge of the primary system operations and does not impose an inordinate amount of assumptions and requirements on the protocols.

A few remarks are in order.

Remark 1: We have assumed that control signals such as ACK, NACK, and CTS will be heard and appropriately interpreted by the nodes in the network. This assumption has been made to allow us to focus on the core issues, and does not fundamentally change the makeup of the problem: For example one may transmit the control messages with higher energy per information bit to secure their decoding, and because control messages have very small entropy this has negligible effect on the overall power budget.

Remark 2: The power constraint has been imposed on a per-block basis for both the primary and secondary. For the primary, this is natural because it does not have CSI and cannot perform long-term power management. The secondary transmits only intermittently, thus for it also an instantaneous power constraint is more natural. The CSI at the secondary is not wasted, however; it has been used to reduce the interference on the primary.

Remark 3: Our discussion concentrates on fixed rate transmission. Joint power and rate allocation may provide some improved performance, but it will also impose further complexity.

Remark 4: Multiple antennas provide additional degrees of freedom to improve the results in this paper. A multiple-inputsingle-output (MISO) secondary can employ a zero-forcing strategy to eliminate the interference seen by the primary receiver, with appropriate CSI [15]. The same solution can be applied with slight modification to our problem to remove the coexistence penalty. We omit the details for brevity.

Remark 5: If the power of the primary is high enough, retransmissions are rare. This implies that the rate delivered to the secondary user will be bursty, therefore the proposed algorithms are suitable for secondaries that can accept a bursty transmission. We use this opportunity to emphasize that we do not consider the proposed methods as a replacement for spectrum sensing, or even necessarily as stand-alone methods, but rather as methods that can perhaps be used in combination with other cognitive protocols. Integration of spectrum sensing protocols with protocols that operate when the primary pair is active is sometimes called generalized cognitive radio [14].

Remark 6: The protocols presented in the paper can work for multiple rounds of primary retransmissions as long as the secondary user over hears the handshake signaling of the primary user. The secondary transmitter has two options based on the system model assumptions. It can transmit only during the primary's first retransmission round and thus allows subsequent primary retransmissions to be interference-free. The other option is to transmit a new packet at each retransmission round of the primary user, since ARQ mechanism is assumed not to be available for the secondary user, with minor modifications to the power control calculations.

\section{CONCLUSION}

This work proposes several protocols for the coexistence of primary and secondary pairs of wireless nodes under fading conditions. The structure of primary ARQ transmissions is exploited to provide nontrivial rate for the secondary user while minimizing the impact on the primary user. The proposed protocols make use of power control, relaying, interference cancellation and multi-user diversity. The protocols are analyzed and their performance is numerically verified. The proposed protocols produce attractive results without imposing any overly restrictive assumptions on the system. They have the further practical advantage of using well-known and wellunderstood system components.

\section{REFERENCES}

[1] J. M. Peha, "Approaches to spectrum sharing," IEEE Commun. Mag., vol. 43, no. 2, pp. 10-12, Feb. 2005.

[2] _ - "Sharing spectrum through spectrum policy reform and cognitive radio," Proc. IEEE, vol. 97, no. 4, pp. 708-719, Apr. 2009.

[3] Q. Zhao and B. M. Sadler, "A survey of dynamic spectrum access," IEEE Signal Process. Mag., vol. 24, no. 3, pp. 79-89, May 2007.

[4] R. Tandra and A. Sahai, "SNR walls for signal detection," IEEE J. Sel. Topics Signal Process., vol. 2, no. 1, pp. 4-17, Feb. 2008.

[5] A. Carleial, "Interference channels," IEEE Trans. Inf. Theory, vol. 24, no. 1, pp. 60-70, Jan. 1978.

[6] N. Devroye, P. Mitran, and V. Tarokh, "Achievable rates in cognitive radio channels," IEEE Trans. Inf. Theory, vol. 52, no. 5, pp. 1813-1827, May 2006.

[7] A. Goldsmith, S. A. Jafar, I. Maric, and S. Srinivasa, "Breaking spectrum gridlock with cognitive radios: an information theoretic perspective," Proc. IEEE, vol. 97, no. 5, pp. 897-914, May 2009.

[8] M. Gastpar, "On capacity under receive and spatial spectrum-sharing constraints," IEEE Trans. Inf. Theory, vol. 53, no. 2, pp. 471-487, Feb. 2007.

[9] A. Ghasemi and E. S. Sousa, "Fundamental limits of spectrum-sharing in fading environments," IEEE Trans. Wireless Commun., vol. 6, no. 2, pp. 649-658, Feb. 2007.

[10] L. Musavian and S. Aissa, "Capacity and power allocation for spectrumsharing communications in fading channels," IEEE Trans. Wireless Commun., vol. 8, no. 1, pp. 148-156, Jan. 2009.

[11] Y. Chen, G. Yu, Z. Zhang, H. Chen, and P. Qiu, "On cognitive radio networks with opportunistic power control strategies in fading channels," IEEE Trans. Wireless Commun., vol. 7, no. 7, pp. 2752-2761, July 2008.

[12] R. Zhang, "Optimal power control over fading cognitive radio channel by exploiting primary user CSI," in Proc. IEEE Global Telecommunications Conference 2008., Nov. 2008, pp. 1-5.

[13] R. Zhang, S. Cui, and Y.-C. Liang, "On ergodic sum capacity of fading cognitive multiple-access and broadcast channels," IEEE Trans. Inf. Theory, vol. 55, no. 11, pp. 5161-5178, Nov. 2009.

[14] O. O. Koyluoglu and H. El Gamal, "On the utility of frequency reuse in cognitive radio channels," in Proc. IEEE International Symposium on Information Theory (ISIT), June 2007, pp. 2161-2165.

[15] R. Zhang and Y.-C. Liang, "Exploiting multi-antennas for opportunistic spectrum sharing in cognitive radio networks," IEEE J. Sel. Topics Signal Process., vol. 2, no. 1, pp. 88-102, Feb. 2008.

[16] K. Eswaran, M. Gastpar, and K. Ramchandran, "Bits through ARQs: spectrum sharing with a primary packet system," in Proc. IEEE International Symposium on Information Theory (ISIT), June 2007, pp. 2171-2175.

[17] L. H. Ozarow, S. Shamai, and A. D. Wyner, "Information theoretic considerations for cellular mobile radio," IEEE Trans. Veh. Technol., vol. 43, no. 2, pp. 359-378, May 1994.

[18] M. Katz and S. Shamai, "Transmitting to colocated users in wireless ad hoc and sensor networks," IEEE Trans. Inf. Theory, vol. 51, no. 10, pp. 3540-3563, Oct. 2005.

[19] W. Wu, S. Vishwanath, and A. Arapostathis, "Capacity of a class of cognitive radio channels: interference channels with degraded message sets," IEEE Trans. Inf. Theory, vol. 53, no. 11, pp. 4391-4399, Nov. 2007. 
[20] A. Jovicic and P. Viswanath, "Cognitive radio: an information-theoretic perspective," in Proc. IEEE International Symposium on Information Theory (ISIT), July 2006, pp. 2413-2417.

[21] — "Cognitive radio: an information-theoretic perspective," IEEE Trans. Inf. Theory, vol. 55, no. 9, pp. 3945-3958, Sep. 2009.

[22] P. Grover and A. Sahai, "Writing on Rayleigh faded dirt: a computable upper bound to the outage capacity," in Proc. IEEE International Symposium on Information Theory (ISIT), June 2007, pp. 2166-2170.

[23] G. Caire, G. Taricco, and E. Biglieri, "Optimum power control over fading channels," IEEE Trans. Inf. Theory, vol. 45, no. 5, pp. 14681489, July 1999.

[24] A. J. Goldsmith and P. P. Varaiya, "Capacity of fading channels with channel side information," IEEE Trans. Inf. Theory, vol. 43, no. 6, pp. 1986-1992, Nov. 1997.

[25] R. Knopp and P. A. Humblet, "Information capacity and power control in single-cell multiuser communications," in Proc. IEEE International Conference on Communications (ICC), vol. 1, June 1995, pp. 331-335.

[26] D. Tse and P. Viswanath, Fundamentals of Wireless Communication. New York: Cambridge University Press, 2005.

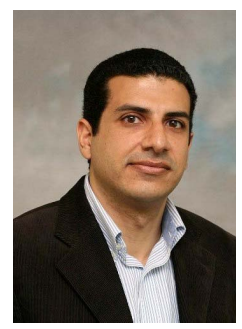

Ramy Abdallah Tannious received the B.S. and M.S. degrees from Alexandria University, Egypt, in 1999 and 2003, respectively, and the Ph.D. degree from the University of Texas at Dallas, TX, in 2008, all in Electrical Engineering. His dissertation work concerned the performance limits of communications in several wireless relay network architectures. From 2000 to 2001, he served in the Signal Corps of the Egyptian Armed Forces. From 2001 to 2008, he held appointments at Alexandria University, Sidpec Petrochemicals, University of South Florida, the University of Texas at Dallas, and Qualcomm Inc. Since 2009, he has been with the University of California Davis as a Post-doctoral Research Scientist in the Department of Electrical and Computer Engineering. His research interests lie in the broad area of wireless communication theory, with applications to relay networks and cooperative communications, cognitive radio networks, and body area networks. He has published over 15 journal and conference papers in these areas.

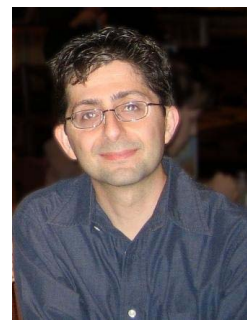

Aria Nosratinia (S'87,M'97,SM'04,F'10) is with the Department of Electrical Engineering at the University of Texas at Dallas, where he is a Jonsson School Distinguished Professor and the director of the Multimedia Communications Laboratory. He received his Ph.D. in Electrical and Computer Engineering from the University of Illinois at UrbanaChampaign in 1996. He has held visiting appointments at Princeton University, Rice University, and UCLA. His interests lie in the broad area of information theory and signal processing, with applications in wireless communications. His recent work has been on cooperative and opportunistic communication and cross-layer issues in communications. $\mathrm{He}$ currently serves as editor for the IEEE TRANSACTIONS ON INFORMATION THEORY, and IEEE TRANSACTIONS ON WiRELESS COMMUNICATIONS, and serves as an officer and member of the Board of Governors of the IEEE Information Theory Society. He has also been an editor for the IEEE Transactions on IMAge Processing, IEEE Signal Processing LeTTERS, IEEE Wireless COMMUNiCATIONS MAGaZine, and JouRnal of Circuits, Systems, AND COMPUTERS. He has been a recipient of the National Science Foundation career award, and is a registered professional engineer in the state of Texas. He was elected Fellow of IEEE for contributions to multimedia and wireless communication 